\title{
Update onarsenic Exposure Link inCancer
}

\author{
MurtazaMustafa $^{1}$, RK.Muniandy ${ }^{2}$, EM.Ellzam ${ }^{3}$, HM.Iftikar $^{4}$, K.Fairrul ${ }^{5}$, \\ ${ }^{*}$ MK Nang ${ }^{6}$, AM.Sharifa ${ }^{7}$, \\ ${ }^{1,2,5.6}$ Faculty of Medicine and Health Sciences, University Malaysia Sabah ,Kota Kinabalu, Sabah, Malaysia \\ ${ }^{3}$ Clinic Family Planning Association, KotaKinabalu, Sabah, Malaysia \\ ${ }^{4}$ Department of Orthopedic, Management And Science University, Selangor, Malaysia \\ ${ }^{7}$ Quality Unit Hospital Queen Elizabeth, KotaKinabalu, Sabah, Malaysia
}

\begin{abstract}
Acute arsenic poisoning is uncommon, but inorganic arsenic exposure is worldwide. The highest affected areas are Bangladesh and West Bengal. Ground water most often gets contaminated due to agriculture and mining activities. Contributory factors include arsenic in the drinking water and through food. Inorganic arsenites(arsenic(III), in drinking water have a much higher acute toxicity than organic arsenates(arsenic(V).Arsenic interferes with cellular longevity by allosteric inhibition of essential metabolic enzyme pyruvate dehydrogenase (PDH) complex, which catalyzes the oxidation of pyruvate to acetyl-CoA by NAD.The common symptoms of arsenic poisoning include headache confusion, severe diarrhea, anddrowsiness. Acute symptoms may include diarrhea, vomiting, vomiting blood, blood in urine, and convulsions, Body organs affected are the lungs,skin, kidneys and liver, and eventuallycoma and death. Arsenic increases the risk of cancer, heart disease, stroke, chronic lower respiratory diseases and diabetes. Arsenic may be measured in blood, urine hair, and finger nails. DMSA monoesters are promising antidotes for arsenic poisoning.
\end{abstract}

Key Words: Arsenicpoisoning, Arsenicassociated cancer, Diagnosis, Therapy.

\section{Introduction}

Arsenic exposure can lead to arsenic poisoning a medical condition that occurs due to elevated levels of arsenic in the body[1].Worldwide more than 200 million people are exposed to higher levels of arsenic. The areas most affected are Bangladesh and West Bengal. The acute poisoning is uncommon [2].The toxicity of arsenic has been described as far as $1500 \mathrm{BC}$ in the Ebers papyrus [3].Arsenic poisoning has been reported throughout the human history. Many noteworthy cases include Francesco (Italy,1541-1587)[4],King Eric XIV(Sweden,1533-1577)[5],King George III's(Great Britain,1738-1820)[6],Napoleon Bonaparte(France,17691821)[7],Charles Francis Hall(USA,1821-1971)[8],Guangxu Emperor(People's Republic of China,18711908)[9],Anderson Mazoka(Zambia,1932)[10].and King FaisaI I(Iraq,1933)[11].Contributory factors include groundwater most often becomes contaminated may also occur from mining or agriculture, exposure to toxic waste sites and traditional medicine[12].Symptoms of brief exposure may include vomiting, abdominal pain, encephalopathy, and watery diarrhea that contain blood. Long- term exposure can result in thickening of skin, darker skin, abdominal pain, diarrhea, heart disease, numbness, and cancer [12].Arsenic increases the risk of cancer[13].Exposure is related to skin, lung,liver, and kidney cancer among others[12].There is no specific treatment for long- term poisoning[1].For acute poisoning treating dehydration is important[1]. Dimercaptosuccinicacid(DMSA) or dimercaptopropane sulfonate(DMPS) may be used while dimercaprol (BAL) is not recommended[14].Hemodialysis may also be used[1]..Prevention is by using the water that does not contain high levels of arsenic [12].The paper provides an overview of chronic exposure to inorganic arsenic and its link in cancer.

\section{Historical Perspectives}

In addition to its presence as a poison, for centuries arsenic was used medicinally. It has been used for over 2,400 years as a part of traditional Chinese medicine [15].Arsenic became a favored method for murder of middle Ages and Renaissance, particularly among ruling classes in Italy allegedly. Because the symptoms are similar to those of cholera, which was common at that, arsenic poisoning often went undetected[7].By the $19^{\text {th }}$ century, it had acquired the nickname "inheritance powder", perhaps because impatient heirs were known or suspected to use it to ensure or accelerate their inheritance[16].In ancient Korea, and particularly in Joseon Dynasty, arsenic- sulfur compounds have been used as a major ingredient of sayak, which was a poison cocktail in capital punishment of high-profiler political figures and members of royal family[16].

Prominent cases: Recent forensic evidence discovered by Italian scientists suggest that Francesco(1541-1587) and his wife were poisoned by his brother and successor Ferdinando[4].The body of Eric XIV(1533-1577) king of Sweden was exhumed in 1958 and modern forensic analysis revealed evidence of lethal arsenic poisoning. 
His last meal was a poisoned bowl of pea soup[5,]. King George III's(1738-1820) personal health was a concern throughout his reign. He suffered from periodic episodes of physical and mental illness. In 1969, researchers asserted that the episodes of madness and other physical symptoms were characteristic of disease porphyria, which was also identified in members of his immediate family and extended family. In addition, a 2004 study of samples of the King's hairs revealed extremely high levels of arsenic, which is a possible trigger of disease symptoms [6].It has been suggested that Napoleon Bonaparte (1769-1821) suffered and died from arsenic poisoning during imprisonment on the island of Saint Helena. Forensic samples of his hair did show high levels 13 times the normal amount, of the element. This however, does not prove deliberate poisoning by Napoleon's enemies: copper arsenide has been used as pigment in some wall papers, and microbiological liberation of the arsenic into the immediate environment would be possible [7].American explorer Charles Francis Hall(18211871) died unexpectedly during his third Arctic expedition abroad the ship Polaris[8,].In 1968 Hall's body was exhumed, and tissue samples of bone, fingernails and hair showed that Hall died of poisoning from large doses of arsenic in last two weeks of his life[17],consistent with the symptoms party members reported. It is possible that he was murdered by Bessels or one of the party members of the expedition [18].In 2008, testing in the People's Republic of China confirmed that GuangxuEmperor(1871-1908) was poisoned with a massive dose of arsenic; suspects include his dying aunt, Empress Dowager Cixi, and her strongman, Yuan Shikai[9].The popular opposition leader in Zambia,AndersonMazoka,whose health deteriorated after the 2001 presidential election.His daughter confirmed after his death on 24 May 2006 the arsenic was found in his body after he died from kidney complications [10].

\section{Contributory Factors inArsenic Poisoning}

Organic arsenic is less harmful than inorganic arsenic. Seafood is a common source of less toxic organic arsenic in the form of arsenobetaine.The arsenic reported in 2012 in fruit juice and rice by Consumer Reports was primarily inorganic arsenic [19].

Arsenic in drinking water: Chronic arsenic poisoning results from drinking contaminated well water over long period of time Manyaquifiers contain high concentration of arsenic salts[20].The World Health Organization(WHO) recommends a limit of $0.01 \mathrm{mg}(10$ parts per billion) of arsenic in drinking waterThis recommendation was established based on the limit of detection for most laborites testing equipment at the time of publication of the WHO water quality guidelines. More recent findings show that consumption of water with levels as low as $0.00017 \mathrm{mg} / \mathrm{L}(0.17$ part per billion) over long periods of time can lead arsenicosis[21].A Chinese study in 1988, the US protection agency quantified the lifetime exposure of arsenic in drinking water at concentrations of $0.001 \mathrm{mg} / \mathrm{L}, 0.00017 \mathrm{mg} / \mathrm{L}$, and $0.000017 \mathrm{mg} / \mathrm{L}$ are associated with a life skin cancer risk of 1 in 10,000,1 in 100,000 and 1 in 1,000,000 respectively. WHO asserts that a level of $0,01 \mathrm{mg} / \mathrm{L}$ poses a risk of 6 in 10,000 chance of life time risk and contends that this level of risk is acceptable[22]]. One of the worst incidents of arsenic poisoning via well water occurred in Bangladesh, which the WHO called the " largest poisoning of a population in history"[23].Taiwanese Blackfoot disease is caused by arsenic contamination of wells, resulting in peripheral vascular disease[24].Mining techniques such as hydraulic fracturing may mobilize arsenic in groundwater and aquifers due to enhanced methane transport and resulting changes in redox conditions and inject fluid containing additional arsenic[25,26].

Exposure by food:It has been found that rice is particularly susceptible to accumulation of arsenic from soil [27].Rice grown in U.S.has an average 260ppb of arsenic, according to a study, but U.S. arsenic remains far below WHO recommended limits[28]. China has set a standard for arsenic limits in food(15ppb)[29] as levels in rice exceeds those in water[30].Arsenic is an ubiquitous element present in American drinking water[31].In the United States levels of arsenic that are above natural levels, but still well below danger level set in Federal safety standards, have been detected in commercially grown chickens[32].The source of arsenic appears to be the food additive roxarsone and nitrasone, which are used to control the parasitic infection coccidiosis as well to increase weight and skin coloring[33].High levels of inorganic arsenic were reportedly found in 83 California wines in 2015[34].Subacute arsenic poisoning caused by ingestion of an arsenic contaminated beer is associated with cardiomyopathy and cardiac failure[35].

\section{Pathophysiology}

Arsenic interferes with cellular longevity by allosteric inhibition of essential metabolic enzyme pyruvate dehydrogenase (PDH) complex, which catalyzes the oxidation of pyruvate to acetyl-CoA by $\mathrm{NAD}^{+}$. With enzyme inhibited, the energy system of the cell is disrupted resulting in a cellular apoptosis episode. Biochemically, arsenic prevents use of thiamine resulting in a clinical picture resembling thiamine deficiency. Poisoning with arsenic can raise lactate levels and lactic acidosis. Low potassium levels in the cells increases the risk of experiencing a life threatening heart rhythm problem from arsenic trioxide. Arsenic in cells clearly 
stimulates the production of hydrogen peroxide $\left(\mathrm{H}_{2} \mathrm{O}_{2}\right)$. Ehen the $\mathrm{H}_{2} \mathrm{O}_{2}$ reacts with certain metals such as iron or manganese it produces a highly reactive hydroxyl radical. Inorganic arsenic trioxide found in ground water particularly affects voltage-gated potassium channels [36],disrupting cellular electrolytic function resulting in neurological disturbances; cardiovascular episodes such as prolonged QT interval (interval on an ECG that contains deflections by ventricular contractions), neutropenia, high blood pressure, central nervous system dysfunction, anemia, and death[37].Arsenic exposure plays a key role in the pathogenesis of vascular endothelial dysfunction as it inactivates endothelial nitic oxide synthase, leading to reduction in the generation and bioavailability of nitric oxide. In addition, the chronic arsenic exposure induces high oxidative stress, which may affect the structure and function of cardiovascular system. Further, the arsenic exposure has been noted to induce atherosclerosis by increasing the platelet aggregation and reducing fibrinolysis. Moreover arsenic exposure may cause arrhythmia by increasing the QT interval and accelerating cellular calcium overload. The chronic exposure to arsenic upregulates the expression of tumor necrosis factor -alpha, interleukin-1,vascular adhesion molecule and vascular endothelial growth factor to induce cardiovascular pathogenesis[38].Tissue culture studies have shown that arsenic compounds block both IKr and IKs channels. Arsenic compounds also disrupt ATP production through several mechanisms. These metabolic interferences lead to death from multi organ failure, probably from necrotic cell death, not apoptosis. A Post mortem reveals brick red colored mucosa, due to severe hemorrhage. Although arsenic causes toxicity, it can also play a protective role [39].

Chemical action: Arsenic inhibits not only the formation of acetyl-CoA but also the enzyme succinic dehydrogenase.Arsenate can replace phosphate in many reactions. It is able to form Glc-6-Arsenate in vitro;therefore it has been argued that hexokinase could be inhibited [40].(Eventually this may be a mechanism leading to muscle weakness in chronic arsenic poisoning).In the glyceraldehyde 3-phosphate dehydrogenase reaction arsenate attacks the enzyme bound thioester.The formed 1-aerseno-3-phosphoglycerate is unstable and hydrolyzes spontaneously. This ATP formation in Glycolysis is inhibited while bypassing the phosphoglycerate kinase (Moreover, the formation of 2,3-bisphosphoglycerate in erythrocytes might be affected, followed by a higher oxygen affinity of hemoglobin and subsequently enhanced cyanosis).As shown by Gresser(1981),sub mitochondrial particles synthesize adenosine-5'-diphosphate-arsenate from ADP and arsenate in presence of succinate. Thus, by a variety of mechanisms arsenate leads to an impairment of cell respiration and subsequently diminished ATP formation [41].Experiments demonstrated enhanced arterial thrombosis in rat animal model, elevation of serotonin levels, thromboxane $\mathrm{A}[2]$ and adhesion proteins in platelets, while human platelets showed similar responses[42].The effect on vascular endothelium may eventually be mediated by arsenicinduced formation of nitric oxide. It was demonstrated that +3 As concentrations substantially lower than concentrations required for inhibition of lysosomal protease cathepsin L in B cell line TA3 were sufficient to trigger apoptosis in same B cell line, while latter could be mediating immunosuppressive effects [43].

Chemico-kinetics:The two forms of inorganic arsenic, reduced(trivalent AS(III)) and oxidized (pentavaleAsV), can be absorbed, and accumulated in tissues and body fluids[43].In the liver,the metabolism of arsenic involves enzymatic and non-enzymatic methylation,the most frequently excreted metabolite $(\geq 90 \%)$ in the urine of mammals is dimethylarsinic acid or cacodylicacid,DMA(V)[44].Dimethlyarsenic acid also known as Agent Blue and was used as herbicide in the American war in Vietnam[44].Arsenic, especially +3 As, binds to single, but with higher affinity to vicinal sulfhydryl groups, thus reacts with a variety of proteins and inhibits their activity. It was also proposed that binding of arsenite at nonessential sites might contribute to detoxification [45].Arsenite inhibits members of the disulfide oxidoreductase family like gluthione reductase[46], and thioredoxin reductase[46].The remaining unbound arsenic $(\leq 10 \%)$ accumulates in cells, which over time may lead to skin,bladder,kidney,liver,lung, and prostate cancers[44].Other forms of arsenic toxicity in humans have been observed in blood, bonemarrow, cardiac, central nervous system, gastrointestinal ,gonadal,kidney,liver,pancreatic,and skin tissues[44].

Arsenic associated with Oxidative stress: Studies have demonstrated that oxidative stress generated by arsenic may disrupt the signal transduction pathways of nuclear transcriptional factors PPAR's,AP-1, and NF-kB[44,47] as well as the pro-inflammatory cytokines IL-8 and TNF- $\alpha[44,47]$.The interference of oxidative stress with signal transduction pathways may affect physiological processes associated with cell growth,metabolic syndrome X, glucose homestasis,lipidmetabolism,obesity,insulin resistance, inflammation, and diabetes2[48].Recent scientific evidence has elucidated the physiological roles of the PPAR's in the $\phi$-hyroxylation of fatty acids and the inhibition of pro-inflammatory transcription factors(NF-kB and AO-1),pro-inflammatory cytokines(IL-!-6-8-12, and TNF- $\alpha$ ),cell4 adhesin molecules(ICAM-1 and VCAM),inducible nitric oxide synthase,oro-inflammatory nitric oxide(N0), and anti-apoptotic factors[44].Epidemiological studies have suggested a correlation between chronic consumption of drinking water contaminated with arsenic and the incidence of Type-2 diabetes [44].The human liver after exposure to therapeutic drugs may exhibit hepatic 
non0cirrhotic portal hypertension, fibrosis, andcirrhosis [44].However, literature provides insufficient evidence to show cause and effect between arsenic and the onset of diabetes mellitus Type 2[44].

\section{Clinical Manifestations}

Frequently symptoms of arsenic poisoning begin with headache confusion, severe diarrhea, and drowsiness. As the the poisoning develops, convulsions and changes in fingernail pigmentation called leukonychia striata(Mee'slines,or Aldrich-Mee's lines) may occur[49]. When the poisoning becomes acute, symptomsmay include diarrhea, vomiting, vomiting blood, blood in the urine, cramping muscles, hair loss, stomach pain, and more convulsions. The organs of the body that are usually affected by arsenic poisoning are the lungs ,skin, kidneys, and liver[50].Finally the arsenic poisoning results in coma and death [51].Arsenic is related to heart disease(hypertension-related cardiovascular disease),cancer[52,53],stroke(cardiovascular disease),chronic lower respiratory diseases and diabetes[54,55].Islam RM and associates in a series of 1682 subjects, suggest an association between chronic arsenic exposure through drinking water and type 2 diabetes(T2D).Risks are generally higher with longer duration of arsenic exposure[56].Chronic exposure to arsenic is related to vitamin to vitamin A deficiency, which is related to heart disease and night blindness[57].Inorganic arsenites(arsenic (III)) in drinking water have a much higher acute toxicity than organic arsenates(arsenic V)[58,wp,16].The acute minimal lethal dose of arsenic in adults is estimated to be 70 to 200 $\mathrm{mg}$ or $1 \mathrm{mg} / \mathrm{kg} /$ day[59].

Arsenic link in Cancer:Arsenic increases the risk of cancer [13].Exposure is related to skin, lung, liver, and kidney cancers among others[12].Its computagenic effects may be explained by interference with base and nucleotide excision repair, eventually through interaction with zinc finger structures[60].Dimethlyarsinicacid,DMA(V), caused DNA single stand breaks resulting from inhibition of repair enzymes at levels of 5 to $100 \mathrm{mM}$ in human epithelial type 11 cells[61].MMA(III) and DMA(III) were also known to be directly genotoxic by effectuating scissions in supercoiled alp X174 DNA[62].Increased arsenic exposure is associated with an increased frequency of chromosomal aberrations[63],micronuclei and sisterchromatid exchanges[64].An explanation for chromosomal aberrations is the sensitivity of the protein tubulin and the mitotic spindle to arsenic. Histological observations confirm effects on cellular integrity, shape and locomotion [65].Research studies on DNA methylation suggest interaction of As with methyltransferases which leads to an inactivation of tumor suppressor genes through hyper-methylation, others state that hypomethylation might occur due to a lack of SAM resulting in aberrant gene activation [66].An experiment by Zhong et al(2001) with arsenite-exposed human lung A549,kidney UOK123,UOK109 and UOK 121 cells isolated eight different DNA fragment by methylation-sensitive arbitrarily primed PCR[67].It turned out that six of the fragments were-layer and two of them were hypo-methylated[67]..Higher levels of DNA methyltransferase mRNA and enzyme activity were found[67].Kitchin (2001) proposed a model of altered growth factors which lead to cell proliferation and thus to carcinogenesis[68].From observations, it is known that chronic low-dose arsenic poisoning can lead to increased tolerance to its acute toxicity[13].MRP!overexpressing lung tumor GLC4/Sb 30-cells poorly accumulate arsenite and arsenate. This mediated through MRP-1 depend efflux[69].The efflux requires GSH,but no As-GSH complex formation[70].Although many mechanisms have been proposed, no definite model can be given for the mechanisms of chronic arsenic poisoning. The prevailing events of toxicity and carcinogenesis might be quite tissue-specific. Current consensus on mode of carcinogenesis is that it acts primarily as a tumor promoter. Its co-carcinogenicity has been demonstrated in several models. However, the finding of several studies that chronically arsenic-exposed Andean populations(as most exposed to UV-light) do not develop skin cancer with arsenic exposure, is puzzling [71].

\section{Diagnosis and Therapy}

Arsenic may be measured in blood or urine to monitor excessive environmental or occupational exposure, confirm a diagnosis of poisoning in hospitalized victims or to assist in the forensic investigation in a case of fatal over dosage. Some analytical techniques are capable of distinguishing organic from inorganic forms of the element. Organic arsenic compounds tend to be eliminated in the urine in unchanged form, while inorganic forms are largely converted to organic arsenic compounds in the body prior to urinary excretion. The current biological exposure index for U.S.workers of $35 \mathrm{ug} / \mathrm{L}$.total urinary arsenic may easily be exceeded by a healthy person eating a seafood meal [72].Tests are available to diagnose poisoning by measuring arsenic in blood,urine, hair, and finger nails. The urine test is the most reliable test for arsenic exposure within the last few days.Urine testing to be done within 24-48 hours for an accurate analysis of an acute exposure. Tests on hair fingernails can measure exposure to high levels of arsenic over the past 6-12 months. These tests can determine if one has been exposed to above average levels of arsenic. They cannot predicthowever, whether the arsenic levels in the body will affect health [73].Chronic arsenic exposure can remain in the body system for a longer 
period of time than a shorter term or more isolated exposure and can be detected in a longer time frame after the introduction of the arsenic, important in trying to determine the source of the exposure [73].Hair is a potential bio indicator for arsenic exposure due to its ability to store trace elements from blood. Incorporated elements maintain their position during growth of hair.Thus for a temporal estimation of exposure, an assay of hair to be carried out with a single hair which is not possible with older techniques requiring homogenization and dissolution of several strands of hairs. This type of biomonitoring has been achieved with newer micro analytical techniques like Synchrotron radiation based X ray fluorescence (SXRF) spectroscopy and Micro particle induced X ray emission (PIXE).The highly focused and intense beams study small spots on biological samples allowing analysis to micro level along with the chemical speciation. In a study, this method has been used to follow arsenic level during and after treatment with Asenious oxide in patients with Acute PromyelocyticLeukemia[74].

Therapy and complementary medicine:Dimercaprol and dimercaptosuccinic acid are chelating agents that sequester the arsenic away from blood proteins and are used and are used in treating acute arsenic poisoning. The most important side effect is hypertension.Dimercaprol is considerably more toxic than succimer [75].DMSA monoesters,e.g.MIADMSA,are promising antidotes for arsenic poisoning. Calcium sodium edetate is also used [76].Supplemental potassium decreases the risk of experiencing a life threatening heart rhythm problem from arsenic trioxide [77]

\section{Conclusions}

Ground water contaminated with arsenic is associated with risk of skin, lung liver, and kidney cancer. At present there is no effective treatment for long term arsenic poisoning. Dimercaptosuccinicacid(DMSA) is useful .Drinking water without high levels of arsenic are the only prevention.

\section{References}

[1]. VahidniaA,van der VoetGB,de Wolff FA.Arsenic neurotoxicity A review.Human\& Experimental Toxicology.2007;26(10):823-32.

[2]. NaujokasMF,AndersonB,AhsanHabibul,etal.The Broad Scope of Health Effects from Chronic Arsenic Exposure:Update on a Worldwide Health Problems.Envir Health Persp.2013;121(3):295-302.

[3]. Howie Frank.Care and conservation of Geological MaterialsRoutlege.p.135.ISBN 9781135385217.

[4]. Mari F,PoletiniA,LippiD,etal.The mysterious death of Francesco 1 de'Medei and Bianca Cappello:an arsenic murder?.BMJ.2006;333(7582):1299-301.

[5]. Ericson Lars(2004).John III:enbiografi(in Swedis).Lund Historiska media.p.109.ISBN 91-85057-47-9.

[6]. King George III:Mad or misunderstood ?.BBC News.2004-07-13.Retrieved

[7]. James G Whorton.(2011).The Arsenic Century.Oxford University Press.ISBN 978-0-19-960599-6.

[8]. MowatFarley.The Polar Passion.The Quest for North Pole.Toronto:McCelland and Stewart Limited,1967,p.124.

[9]. Forensic scientists:China's reformist second-to-last emperor was murdered.(http://news.xinhuanet.com/english/2008-11/03/ content 1030 1467. htm) Xinhua,November 3,2008

[10]. MutinaMazokaMmembe-Young foused\& forward thinking.Commerce gazette .Retrived 28 December 2016.

[11]. Mohammed AI Janabi,78 years after the murder of King Faisal the first, Iraq Law Network.http://www..qanon302.net/news/news.php"actions=view \&id=7230.

[12]. RatnaikeRN.Acute and chronic arsenic toxicity.Postgrad Med J.2003;79(933):391-96.

[13]. Gebel TW.Genotoxicity of arsenical compounds.Int J HygEnvir Health. 2001;203(3):249-62.

[14]. Andersen 0le Aaseth.A review of pitfalls and progress in chelation treatment of metal poisoning.J Trace Elemnt MedBiol.2016;38:74-80.

[15]. Application of arsenic trioxide for the treatment of lupus arthritis.Chinese Medical Association..

[16]. MBC NEWS.

[17]. Fleming Fergus.”Ninety Degrees North". The Quest for the North Pole.Toronto:McClelland and Stewart Limited.1967,p.124.

[18]. Chauncey Loomis."Charles Francis Hall 1821-1871"(PDF).

[19]. Arsenic in your food: Our findings show a real need for federal standards for this toxin. Consumer Reports. November 2012.Retrieved 22 November 2012.

[20]. WHO Water-related diseases (Http://who.int/water_sanitation_health/diseases/arseniccosis/en/)

[21]. Concentration of selected toxic metals in groundwater and some cereals grown in Shibgani area of Chapai Nawabganj, Rajshahi, Bangladesh (http:// www.currentscience.ac.in/Volumes/101/03/0427.pdf)(p.429)Current Science Journal,retrieved August 29,2014.

[22]. Towards an assessment of the socioeconomic impact of arsenic poisoning in Bangladesh:Health effects of arsenic in drinking water(p.5)(PDF).Drinking water Quality.WHO.Retrieved 2014.

[23]. Contamination of drinking- water by arsenic in Bangladesh: a public health emergency.(PDF). World Health Organization.Retrieved 2013-08-27.

[24]. TsenfCH,etal.Long-term arsenic exposure and ischemic heart disease in arseniasis-hyperendermic village in Taiwan.Toxicol Lett.2003;137:15[PIMD:12505429].

[25]. Brown RA,KatrinaA,PattersonKE,etal.Attenuation of Naturally Occuring Arsenic at Petroleum Hydrocarbon-Impacted Sites.(Http:www.api.org/-/media/

EHS/Clean_Water/Ground Water_Quality/Battlele_2010_API_Arsenic_Mngnt.ashx).www.battlle.org/Chlorcon.

[26]. MurcottS(2012).Arsenic contamination in the World.London:IWA Publishing.

[27]. Deborah Kotz(Dec.8.2011).Do you need to worry about arsenic in rice.BostonGlobe.Retrieved December 8,2011.

[28]. Surprisingly high concentrations of toxic arsenic species found in U.S.rice.

[29]. "Rice as a source of arsenic exposure".

[30]. China Inorganic in Rice-An Underestimated Health Threat

[31]. USGS NAWQA Arsenic in Groundwater. 
[32]. Study Finds an Increase in Arsenic Levels in Chicken:.New York Times.May 11.2013.

[33]. FDA:Pfizer will voluntarily suspend sale of animal drug 3-Nitro.

[34]. Dangerous arsenic levels found in California wine from 28 producers, suit claims.New York Daily News March $21,2015$.

[35]. Hertz PicciottoJ,etal.Does arsenic exposure increase the risk for circulatory disease?.Am J Epidemiol.2000;151:174[PMID $10645820]$.

[36]. Zhou J,WangW,WeiQF,etal.Effects of arsenic trioxide on voltage dependent potassium channels and on cell proliferation ofhuman multiple myeloma cells.Chin Med J.2007;120(14):1266-9.PMID 17697580.

[37]. KonduriGG,BakhutashciliI,EisA,etal.Impaired Voltage Gated Potassium Channel Responses in a Fetal Lamb Model of Persistent Pulmonary Hypertension of Newborn.Pediatric Research. 2009;66(3):289-294.

[38]. BalakumarPitchai,KaurJagdeep.Arsenic Exposure and Cardiovascular disorders:AnOverview.Cardiovascular Toxicology.2009;9(4):169-76.

[39]. KlaassenCurtis,Watkins John. Casarett and Doull's Essentials of Toxicology>McGraw -Hill.p.512.ISBN 978-0-07-138914-3

[40]. HudgeMF.Arsenic and potential mechanisms of actions.Toxicilogy Letters.2002;133(1):1-16.

[41]. Gresser MJ.ADP-arsenate.Formatiion by sunchondrial particles under phosphorylating condition.JBiologChem.1981;256(12):58813.PMID 7240187

[42]. Lee MY,Bae 0N,Chung SM,etal.Enhancement of platelet aggregation and thrombus formation by arsenic in drinking water:acontributing factor to cardiovascular disease.Toxiciology and Applied Phamacol.2002;179(2):83-8.

[43]. HarrissonJW,PackmanEW,AbbottDD.Acute oral toxicity and chemical and physical properties of arsenic trioxides.AMA Archivesof Indust Health.1958;17(2):118-23.

[44]. Vigo JB,EllzeyJT.Effects of Arsenic Toxicity at the Cellular level:ARview.Texas J Microscopy.2006;37(2):45-49.

[45]. AlphosianHV,MaiorinoRM,DartRC,etal.Urinary excretion of meso-2,3-dimercaptosuccinic acid in human subjects.Clin PharmTherpeut.1989;45(5):520-6.

[46]. Rom WN,MarkowitzSB.Environmental Occupational Medicine.Lippincott Williams \&Wilkins.pp.104-5.ISBN 978-0-7817-6299-1.

[47]. $\mathrm{Hu}$ Y,JinX,SnowET.Effects of arsenic on transcription factor AP-1 and NF-kB DNA binding activity and related gene expression.Toxocol Lett.2002;133(1):33-45.

[48]. Kota BP,HuangTH,RoufogalisBD.An overview on biological mechanisms of PPARs.Pharm Res.2005;51(2):85-94

[49]. YalcmTuzum(2009).Leukonchia(PDF).

[50]. Test ID:ASU.Arsenic,24 Hour Urine,ClinicalInformation.Myo Medical Laboratories Catalog.MyoClinic.Retrieved 2012-09-25

[51]. Arsenic Poisoning.IHCWorld.Retrieved 2 May 2014

[52]. Tseng CH,ChongCK,Tseng CP, et al.Long -term arsenic exposure and ischemic heart disease in arseniasis-hyperendemic village in Taiwan..Toxocol Lett. 2003; 137(1-2):15-21.

[53]. Smith AH,Hopenhayn-RischC,BatesMN,etal.Cancer risks from arsenic in drinking water.Environ Health Persepect.1992;97:25967.

[54]. HendryxM,Mortality from heart,respiratory,and kidney disease in coal minning areas of Applachia.Int Arch Occup Environ Health.2009;82(2):243-9.

[55]. Navas-AcienA,SilbergeldEK,Pastor-BarriusoR,etal.Arsenic exposure and prevalence of type 2 diabetes in US adults.JAMA.2008;300(7):814-22.

[56]. Islam MR,IsmailK,HassanNMS,etal.Association between type 2 diabetes and chronic exposure in drinking water:A cross sectional study in Bangladesh.Environ Health.2012;11:38.

[57]. Hsueh YM,WuWL,HuangYL,etal.Low serum carotene level and increased risk of ischemic heart disease related to long-term arsenic exposure .Atherosclerosis. 1998; 141(2):249-57.

[58]. Kingston RL,HallS,SiorisL.Clinical observations and medical outcome in 149 cases of arsenate ant killeringestion.JToxicolClinToxicol.1993;31(4):581-91.

[59]. Dart RC(2004).Medical Microbiology.Philadelphia:William\& Wilkins.pp.1393-1401.ISBN 0-7817-2845-2

[60]. HartwigA,SchwerdtleT.Interactions by carcinogenic metal compounds with DNA repair processes:toxicologicalimplications.Toxicol Lett.2002;127(!-3):47-54.

[61]. Yamanka K, Hayashi H,TachikawaM,etal.Metabolic methylation is a possible genotoxicity-enhancing process of inorganic arsenics.Mutation Research. 1997; 394(1-3):95-101.

[62]. Mass MJ,TennantA,RoopBC,etal.Methylated trivalent arsenic species are genotoxic.ChemResr Toxocol.2001;14(4):355-61

[63]. Maki-PaakanenJ,KurttioP,PaldyA,etal.Association between the clastogenic effect in peripheral lymphocytes and human exposure to arsenic through drinking water.Envirom Mole Mutagen.1998'32(4):301-13.

[64]. Warner ML,MooreLE,SmithMT,et al. Increased micronuclei in exfoliated bladder cells of individuals who chronically ingest arsenic-contaminated water in Nevada.CancerEpidemiolBiomar Preven.1994;3(7):583-90.

[65]. BernstamL,NriguJ.Molecular Aspects of arsenic stress.JToxicol Environ Health Part B Crit Rev.2000;3(4):293-22.

[66]. Goering PL,AposhianHV,MassMJ,etal.The enigma of arsenic carcinogenesis role of metabolism.Toxocol Sci.1999;49(1):5-14.

[67]. ZhongCX,MassMJ.Bothhypomathylation and hypermethylation of DNA associated with arsenic exposure in cultures of human cells identified by methylation-sensitive arbitrarily-primed PCR.Toxicol Lett.2001;122(3):223-34.

[68]. KitchinKT.Recent advances in arsenic carcinogenesis modes of action,animal model systems,and methylated arsenicmetabolites.ToxicolAppl Pharm.2001;172(3):249-61.

[69]. VemhetL,AllainN,BardiauC,etalDifferential sensitivities of MRP1-overexpressing lung tumor cells to cytotoxic metals.Toxocol.2000;142(2):127-34.

[70]. SalemoM,PetroutsaM,GarnierSA.The MRP!-mediated effluxes of arsenic and antimony do not require arsenic-glutathione and antimony complex formation.JBioenergentic Biomembranes.2002;34(2):135-45.

[71]. Gebel T.Confounding variables in the environmental toxicology of arsenic.Toxicol.2000;144(1-3)155-62.

[72]. R Baselt.Disposition of Toxic Drugs and Chemicals in Man, $8^{\text {th }}$ edition,BiochemicalPublications,Foster City,CA,2008,pp.106-110.

[73]. ToxFAQs for Arsenic.Agency for Toxic Substances and Disease Registry.Archieved from the original on 15 January 2009,Retrieved 2009.

[74]. NicolisI.CurisE,DeschampsP,etal.Asenite medicinal use, metabolism, pharmacokinetics and monitoring in human hairs.Biochemie.2009;91(10):1260-7.

[75]. Dimercaprol medical facts from Drugs com.

[76]. [KreppelH,ReichlFX,KleineA,etal.Antodotal efficacy of newly synthesized dimercaptosuccinic acid(DMSA) monoester in experimental arsenic poisoning in mice.Findam.Appl Toxicol.26(2):239-45(1995)].

[77]. Arsenic Trioxide (Trisenox ®). The Abramson Cancer Center of the University of Pennsylvania Last Modified:December 25,2005. 